\title{
Indonesian Adaptation and Psychometric Properties Evaluation of the Big Five Personality Inventory: IPIP-BFM-50
}

\author{
Hanif Akhtar ${ }^{1} \mathcal{E}$ Saifuddin Azwar \\ 1,2Faculty of Psychology, Universitas Gadjah Mada
}

\begin{abstract}
The big five personality traits are the best acknowledged and most commonly used the model in psychology. Therefore, many personality scales are developed based on this personality model. IPIP-BFM-50 is one of the open-source scales measuring big five personalities that is widely used by world researchers. This study is aimed to adapt IPIPBFM-50 into Indonesian and evaluate its psychometric properties. The adaptation was carried out by following the guidelines of the International Test Commission (ITC). The result shows that IPIP-BFM-50 Indonesia has Aiken's V index ranging from $0.71-0.98$. Alpha reliability with $\mathrm{n}=502$ ranges from 0.762 (agreeableness) to 0.862 (emotional stability). Factor analysis shows that the items are well-grouped according to their dimensions with loading factor ranging from 0.34 to 0.75 on the factors measured. Thus, IPIP-BFM-50 Indonesia has satisfactory psychometric properties and can be utilized to measure the Big five personality.
\end{abstract}

Keywords: adaptation, big five; IPIP-BFM-50; reliability; validity

The popularity of big five personality model is currently growing. We tried to trace the Scopus database related to the articles with titles, abstracts, and keywords "Big Five" and "Five Factor Models" for the past five years. As a result, as of January 2018, there were 6357 publications containing titles, abstracts, and keywords "Big Five" and 26,792 publications which contained the title, abstract, and keywords "Five Factor Model". The graphs presented for publications published each year tend to increase. This fact shows that the big five models is still interesting to be investigated. This condition also has implications for the development of personality measures based on the big five personality model. There are two models explaining these models, the Big Five developed by

\footnotetext{
1 Address for correspondence:

hanif.akhtar27@gmail.com; sfazwar@ugm.ac.id
}

Goldberg and the Five-Factor Model model developed by Costa and McCrae. Both of these models are comparable, the minor difference is in naming factors. Although these names are sometimes used interchangeably, they are rooted in two different research traditions. Goldberg's Big Five was developed based on the lexical tradition while Costa and McCrae's Five Factor Models were developed based on the tradition of questionnaire research.

Several instruments based on the big five personality model have been developed. Some of these instruments include the Big Five Inventory (BFI) developed by John \& Srivastava (1999), NEO PI-R developed by Costa \& McCrae (1995), and Triat Descriptive Adjective 
(TDA) developed by Goldberg (1992). Several studies have been conducted to adapt and validate these instruments in various languages and cultural contexts, but unfortunately, some instruments such as NEO PI-R are copyrighted and users are required to purchase.

One of the conveniences in personality measurement is the International Personal Pool (IPIP) which was initiated by several scientists, including Goldberg. IPIP is an international collaboration to develop personality inventories which can be accessed by everyone through the website (Goldberg et al., 2006). All researchers are free to use existing items. At present, there are 3,320 items arranged on several scales. There are already 686 international publications which examine and use the scale from IPIP (http://ipip.ori.org/ index.htm). The scales in IPIP include IPIP Big Five Factor Markers (IPIP-BFM), MiniIPIP, IPIP-NEO, IPIP-16PF, IPIP-HEXACO, and IPIP-VIA. The items in IPIP are items that also measure the same constructs with various commercial measuring instruments, such as IPIP-NEO which measures the same construct as NEO PI-R (Goldberg et al., 2006).

One of the most widely used scales in IPIP is IPIP Big Five Factor Marker (IPIPBFM) used in the Goldberg (1992) study. IPIP-BFM consists of two versions, namely version 50 and 100 items. This study will focus on IPIP-BFM version 50 items which will be called IPIP-BFM-50. IPIP-BFM-50 and contains 50 items in the form of short phrases on a scale of 1-5 that measure big five personality dimensions, namely: 1) Extraversion which shows the level of activity and energy, 2) Agreeableness which shows a positive attitude towards others, 3) Conscientiousness which shows the level of the organization in achieving its objectives and task management, 4)
Emotional stability which shows the level of emotional stability, and 5) Intellect which shows intellectual openness (Strus, Cieciuch, \& Rowiński, 2017).

Studies related to IPIP-BFM-50 have been conducted by many researchers in various purposes, such as adaptation and validation (Gow, Whiteman, Pattie, \& Deary, 2005; Mlačić \& Goldberg, 2007; Strus et al., 2017; Ypofanti et al., 2015) and development of the short version (Donnellan, Oswald, Baird, \& Lucas, 2006; Topolewska, Skimina, Strus, Cieciuch, \& Rowiński, 2017). In Indonesia, research to adapt IPIP-BFM-50 has been conducted by Maharani (2013) in her thesis, but unfortunately, two out of the five scales have reliability below 0.7 . In addition, the results of the factor analysis are also unsatisfactory. Therefore, there needs to be a replication study related to the adaptation process along with a more comprehensive psychometric property report.

In Indonesia, studies related to adaptation and validation of the big five personality measure were also conducted by Ramdhani (2012) who adapted the Big Five Inventory (BFI) developed by John \& Srivastava (1999). However, BFI and IPIPBFM have different characteristics related to the number of items, the name of the factors, and the characteristics of their use. The results of the Ramdhani (2012) study also show that Indonesian BFI still requires improvement because some items have low loading factors. In addition, this instrument has several unfamiliar terms, such as "asertif (assertive)", "estetik (aesthetic)", and "gusar (angry)".

Another advantage of IPIP-BFM is that this measure is open-source, in order that each researcher can use it freely and contribute their research results on the IPIP website. Due to its open source character, many world researchers utilize it for cross- 
cultural research. This is different from BFI, although the utilization of this inventory for non-commercial research is open, the use of other things still requires permission from inventory developers.

We regard the importance of alternative instruments for measuring the big five personality traits in Indonesia which have satisfactory psychometric properties. The existence of IPIP-BFM-50 in the Indonesian language besides being an alternative to the big five personality scale is also a comparable measure for crosscultural studies. Besides that, due to its open-source character, IPIP-BFM Indonesia can also make a positive contribution in the study of personality in the world.

Therefore this study aims to adapt and evaluate the psychometric properties of IPIP-BFM-50. This research is one of the main stand-alone stages of research conducted by researchers to compile a short scale from IPIP-BFM-50. The research will be divided into two phases, the first phase aims to translate IPIP-BFM-50 based on guidelines from the International Test Commission (ITC). While the second stage aims to evaluate psychometric properties by accessing the validity and reliability of the scale.

\section{Method}

\section{Design}

Stage 1: Adaptation of IPIP-BFM-50 into Indonesian

This research was divided into several steps. This step was taken in accordance with the guidelines of the International Test Commission (2016) accompanied by technical considerations from Beaton, Bombardier, Guillemin, \& Ferraz (2000) and (Azwar, 2017). After asking permission from the scale developer, the steps taken in the process of adaptation of IPIP-BFM to
Indonesian were as follows: 1) Translating the scale of IPIP-BFM-50 into Bahasa Indonesia. Translation into Bahasa Indonesia was carried out by two translators independently. Translators were Indonesian citizens who had a minimum IELTS score of 7.00, had a psychology education background, and had ever lived in an English-speaking country; 2) Discussing the translation results of the two translators and obtaining a complete translation draft based on the agreement of the two translators; 3) Backtranslating the translation draft into English by a professional translator. The results of the back-translation were consulted with the scale developer to see whether it was in accordance with the meaning; 4) Reviewing the results of the translation to ensure the equivalence of translations by experts. The expert in this matter was a Psychology lecturer who had experience in translating and had ever lived in an English-speaking country; 5) Testing readability of the scale to see whether instruction and content could be understood by laymen; 6) Test the content validity with Aiken's V method. Evaluation on a scale was related to the relevance of items measuring big five dimensions. It was done by 12 rater people who had an indepth understanding of the big five personality theory. The minimum $\mathrm{V}$ index value set was 0.69 (Aiken, 1985).

Stage 2: Evaluation of psychometric properties of Indonesian IPIP-BFM-50

Evaluation of psychometric properties was conducted by testing the item-total correlation, alpha reliability, and exploratory factor analysis. The minimum value of the item-total correlation received in general according to Azwar (2012) was 0.3, but the standard can be reduced until no less than 0.2. Whereas, according to Kline (1986) the item-total correlation value must 
be above 0.2. We determined the minimum item-total correlation was 0.25 . the reliability test was estimated by internal consistency approach with Alpha Cronbach. The expected value of the reliability coefficient was above 0.70 according to the minimum reliability coefficient suggested by Nunnally (1978) on the instrument for research. If there is an item that has an item-total correlation of less than the minimum limit, then the item must be revised. If all of the criteria had been fulfilled, then factor analysis was carried out.

\section{Subject}

The subjects used for the evaluation of psychometric properties were late adolescents and adults residing in Yogyakarta. The data used for the analysis of item-total correlation and reliability was $n=115$, while the data for factor analysis was $n=502$.

\section{Result}

Stage 1: Adaptation of IPIP-BFM-50 into Indonesian

The results of the research in stage 1 will be presented based on the steps taken, namely as follows. Firstly, we requested permission from the scale developer. Request for permission to adapt IPIP-BFM into Indonesian was conducted in direct correspondence via email with Lewis Goldberg as the administrator of the IPIP website on November 12, 2017. On
November 13, 2017 permission to adapt was granted. After the permission was granted, we translated the scale by two translators independently within one week. The synthesis was conducted to compile final translation results of the two translators facilitated by the researcher. Selection of the appropriate translation results refers to the meaning of words in the Oxford dictionary, word familiarity for Indonesian respondents, and sentence efficiency. Explanation of the translation and synthesis process will be explained based on the personality dimensions below.

\section{Dimension of extraversion}

The translation of the items in the extraversion dimension can be seen in table 1. Items in the extraversion dimension were relatively easy to translate. There were only a few minor differences from the translation of the results of translators $\mathrm{A}$ and B. For example item number 31. Translator A translated the words "parties" as "pesta", while translator B translated the word as "acara". After being discussed, referring to the context of Indonesian people who rarely hold parties, it was decided to translate the words "parties" as "acara".

\section{Dimension of agreeableness}

The translation of the items in the agreeableness dimension can be seen in table 2 . 
Table 1.

Translation of items in the extraversion dimension

\begin{tabular}{|c|c|c|c|c|}
\hline No & Original item & Translation A & Translation B & Final translation \\
\hline E1 & $\begin{array}{l}\text { Am the life of the } \\
\text { party. }\end{array}$ & $\begin{array}{l}\text { Menghidupkan suasana } \\
\text { dan semangat dalam } \\
\text { sebuah pesta pertemuan/ } \\
\text { perkumpulan }\end{array}$ & $\begin{array}{l}\text { Menghidupkan suasana } \\
\text { dalam suatu acara }\end{array}$ & $\begin{array}{l}\text { Menghidupkan suasana } \\
\text { dalam suatu acara }\end{array}$ \\
\hline E6 & Don't talk a lot. & Tidak banyak bicara & Tidak banyak berbicara & Tidak banyak berbicara \\
\hline E11 & $\begin{array}{l}\text { Feel comfortable } \\
\text { around people. }\end{array}$ & $\begin{array}{l}\text { Merasa nyaman berada } \\
\text { di sekitar orang-orang }\end{array}$ & $\begin{array}{l}\text { Merasa nyaman di } \\
\text { sekitar orang lain }\end{array}$ & $\begin{array}{l}\text { Merasa nyaman di } \\
\text { sekitar orang lain }\end{array}$ \\
\hline E16 & $\begin{array}{l}\text { Keep in the } \\
\text { background. }\end{array}$ & Tidak ingin tampil & $\begin{array}{l}\text { Tidak ingin tampil di } \\
\text { depan }\end{array}$ & $\begin{array}{l}\text { Lebih suka bekerja di } \\
\text { belakang layar }\end{array}$ \\
\hline E21 & Start conversations & Memulai percakapan & $\begin{array}{l}\text { Memulai suatu } \\
\text { percakapan }\end{array}$ & $\begin{array}{l}\text { Memulai suatu } \\
\text { percakapan }\end{array}$ \\
\hline E26 & Have little to say. & Tidak banyak berkata & Sedikit berkata & Sedikit berkata \\
\hline E31 & $\begin{array}{l}\text { Talk to a lot of diffe- } \\
\text { rent people at } \\
\text { parties. }\end{array}$ & $\begin{array}{l}\text { Beinteraksi dengan } \\
\text { banyak orang dalam } \\
\text { sebuah pesta }\end{array}$ & $\begin{array}{l}\text { Berinteraksi dengan } \\
\text { banyak orang pada suatu } \\
\text { acara }\end{array}$ & $\begin{array}{l}\text { Berinteraksi dengan } \\
\text { banyak orang dalam } \\
\text { suatu acara }\end{array}$ \\
\hline E36 & $\begin{array}{l}\text { Don't like to draw } \\
\text { attention to myself. }\end{array}$ & $\begin{array}{l}\text { Tidak suka menjadi } \\
\text { pusat perhatian }\end{array}$ & $\begin{array}{l}\text { Tidak suka menjadi } \\
\text { pusat perhatian }\end{array}$ & $\begin{array}{l}\text { Tidak suka menjadi } \\
\text { pusat perhatian }\end{array}$ \\
\hline E41 & $\begin{array}{l}\text { Don't mind being } \\
\text { the center of } \\
\text { attention. }\end{array}$ & $\begin{array}{l}\text { Tidak masalah ketika } \\
\text { menjadi pusat perhatian }\end{array}$ & $\begin{array}{l}\text { Suka menjadi pusat } \\
\text { perhatian }\end{array}$ & $\begin{array}{l}\text { Tidak keberatan menjad } \\
\text { pusat perhatian }\end{array}$ \\
\hline E46 & $\begin{array}{l}\text { Am quiet around } \\
\text { strangers. }\end{array}$ & $\begin{array}{l}\text { Menjadi pendiam saat } \\
\text { berada di sekitar orang } \\
\text { asing/tidak dikenal }\end{array}$ & $\begin{array}{l}\text { Tidak banyak berbicara } \\
\text { pada orang yang tidak } \\
\text { dikenal }\end{array}$ & $\begin{array}{l}\text { Tidak banyak berbicara } \\
\text { pada orang yang tidak } \\
\text { dikenal }\end{array}$ \\
\hline
\end{tabular}

Table 2.

Translation of items in the Agreeableness dimension

\begin{tabular}{|c|c|c|c|c|}
\hline No & Original item & Translation A & Translation B & Final translation \\
\hline A2 & $\begin{array}{l}\text { Feel little concern } \\
\text { for others. }\end{array}$ & $\begin{array}{l}\text { Tidak terlalu peduli } \\
\text { dengan orang lain }\end{array}$ & $\begin{array}{l}\text { Tidak terlalu } \\
\text { memedulikan orang lain }\end{array}$ & $\begin{array}{l}\text { Tidak terlalu } \\
\text { memedulikan orang lain }\end{array}$ \\
\hline A7 & $\begin{array}{l}\text { Am interested in } \\
\text { people. }\end{array}$ & $\begin{array}{l}\text { Memperhatikan/peduli } \\
\text { dengan orang lain }\end{array}$ & Peduli dengan orang lain & $\begin{array}{l}\text { Peduli dengan orang } \\
\text { lain }\end{array}$ \\
\hline A12 & Insult people. & $\begin{array}{l}\text { Kasar terhadap orang } \\
\text { lain }\end{array}$ & $\begin{array}{l}\text { Bersikap kasar pada orang } \\
\text { lain }\end{array}$ & $\begin{array}{l}\text { Bersikap kasar pada } \\
\text { orang lain }\end{array}$ \\
\hline A17 & $\begin{array}{l}\text { Sympathize with } \\
\text { others' feelings. }\end{array}$ & $\begin{array}{l}\text { Bersimpati dengan } \\
\text { perasaan orang lain }\end{array}$ & $\begin{array}{l}\text { Bersimpati dengan } \\
\text { perasaan orang lain }\end{array}$ & $\begin{array}{l}\text { Bersimpati dengan } \\
\text { perasaan orang lain }\end{array}$ \\
\hline $\mathrm{A} 22$ & $\begin{array}{l}\text { Am not interested } \\
\text { in other people's } \\
\text { problems. }\end{array}$ & $\begin{array}{l}\text { Tidak tertarik dengan } \\
\text { masalah orang lain }\end{array}$ & $\begin{array}{l}\text { Tidak tertarik membahas } \\
\text { permasalahan orang lain }\end{array}$ & $\begin{array}{l}\text { Tidak tertarik dengan } \\
\text { masalah orang lain }\end{array}$ \\
\hline A27 & Have a soft heart. & Lemah lembut & Lemah lembut & Lemah lembut \\
\hline A32 & $\begin{array}{l}\text { Am not really } \\
\text { interested in others. }\end{array}$ & $\begin{array}{l}\text { Tidak terlalu tertarik } \\
\text { dengan orang lain }\end{array}$ & $\begin{array}{l}\text { Tidak terlalu tertarik } \\
\text { dengan orang lain }\end{array}$ & $\begin{array}{l}\text { Tidak terlalu tertarik } \\
\text { dengan orang lain }\end{array}$ \\
\hline A37 & $\begin{array}{l}\text { Take time out for } \\
\text { others. }\end{array}$ & $\begin{array}{l}\text { Melakukan sesuatu } \\
\text { untuk orang lain }\end{array}$ & $\begin{array}{l}\text { Mengorbankan waktu } \\
\text { untuk orang lain }\end{array}$ & $\begin{array}{l}\text { Meluangkan waktu } \\
\text { untuk orang lain }\end{array}$ \\
\hline A 42 & $\begin{array}{l}\text { Feel others' } \\
\text { emotions. }\end{array}$ & $\begin{array}{l}\text { Merasakan emosi yang } \\
\text { dirasakan orang lain }\end{array}$ & $\begin{array}{l}\text { Memahami perasaan } \\
\text { orang lain }\end{array}$ & $\begin{array}{l}\text { Memahami perasaan } \\
\text { orang lain }\end{array}$ \\
\hline A 47 & $\begin{array}{l}\text { Make people feel at } \\
\text { ease. }\end{array}$ & $\begin{array}{l}\text { Membuat orang lain } \\
\text { merasa tenang }\end{array}$ & $\begin{array}{l}\text { Membuat orang lain } \\
\text { merasa nyaman }\end{array}$ & $\begin{array}{l}\text { Membuat orang lain } \\
\text { merasa nyaman }\end{array}$ \\
\hline
\end{tabular}


Items in the dimension of agreeableness were also relatively easy to translate. There were only a few minor differences from the translation of the results of translators $\mathrm{A}$ and B. For example item number 37. The item "Take time out for others" by translator A was interpreted as "Melakukan sesuatu untuk orang lain", while translator B translated the word as "Mengorbankan waktu untuk orang lain". From the results of the discussion, it was agreed that "take time out" is more correctly interpreted as "mengorbankan waktu", but the word "mengorbankan" itself was a concern because it was tendentious and prone to social desirability. Therefore it was decided the translation of the item was "Meluangkan waktu untuk orang lain".

\section{Dimension of conscientiousness}

The translation of the items in conscientiousness dimension can be seen in table 3 .

There were several different translations on consientousness dimension items. For example item number 13 . The word "details" by translator A was interpreted as "kecil", while translator B was interpreted as "rinci". In the discussion it was agreed that the word "details" could be interpreted as "kecil", but the word was also ambiguous in Indonesian, because it could be interpreted as a small measure. Referring to the word meaning and word ambiguity, it was finally decided the final translation was "rinci".

Table 3.

Translation of items in the conscientiousness dimension

\begin{tabular}{|c|c|c|c|c|}
\hline No & Original item & Translation A & Translation B & Final translation \\
\hline $\mathrm{C} 3$ & $\begin{array}{l}\text { Am always } \\
\text { prepared. }\end{array}$ & $\begin{array}{l}\text { Selalu mempersiapkan } \\
\text { segala sesuatu }\end{array}$ & $\begin{array}{l}\text { Selalu mempersiapkan } \\
\text { segala hal }\end{array}$ & $\begin{array}{l}\text { Selalu mempersiapkan } \\
\text { segala hal }\end{array}$ \\
\hline $\mathrm{C} 8$ & $\begin{array}{l}\text { Leave my } \\
\text { belongings around. }\end{array}$ & $\begin{array}{l}\text { Meninggalkan barang } \\
\text { pribadi di mana saja }\end{array}$ & $\begin{array}{l}\text { Meninggalkan barang } \\
\text { pribadi di sembarang } \\
\text { tempat/ teledor }\end{array}$ & $\begin{array}{l}\text { Meninggalkan barang } \\
\text { pribadi di sembarang } \\
\text { tempat }\end{array}$ \\
\hline C13 & $\begin{array}{l}\text { Pay attention to } \\
\text { details. }\end{array}$ & $\begin{array}{l}\text { Memperhatikan hal-hal } \\
\text { kecil }\end{array}$ & $\begin{array}{l}\text { Memperhatikan hal-hal } \\
\text { dengan rinci }\end{array}$ & $\begin{array}{l}\text { Memperhatikan hal-hal } \\
\text { secara rinci }\end{array}$ \\
\hline C18 & $\begin{array}{l}\text { Make a mess of } \\
\text { things. }\end{array}$ & Kurang berhati-hati & Mengacaukan banyak hal & $\begin{array}{l}\text { Mengacaukan banyak } \\
\text { hal }\end{array}$ \\
\hline $\mathrm{C} 23$ & $\begin{array}{l}\text { Get chores done } \\
\text { right away. }\end{array}$ & $\begin{array}{l}\text { Segera menyelesaikan } \\
\text { tugas yang diberikan }\end{array}$ & Tidak menunda pekerjaan & $\begin{array}{l}\text { Tidak menunda } \\
\text { pekerjaan }\end{array}$ \\
\hline $\mathrm{C} 28$ & $\begin{array}{l}\text { Often forget to put } \\
\text { things back in their } \\
\text { proper place. }\end{array}$ & $\begin{array}{l}\text { Sering lupa meletakkan } \\
\text { kembali barang di } \\
\text { tempat semula }\end{array}$ & $\begin{array}{l}\text { Sering lupa meletakkan } \\
\text { barang kembali pada } \\
\text { tempatnya }\end{array}$ & $\begin{array}{l}\text { Sering lupa meletakkan } \\
\text { barang kembali pada } \\
\text { tempatnya }\end{array}$ \\
\hline $\mathrm{C} 33$ & Like order. & Menyukai keteraturan & Menyukai keteraturan & Menyukai keteraturan \\
\hline C38 & Shirk my duties. & $\begin{array}{l}\text { Mengabaikan tugas- } \\
\text { tugas yang diberikan }\end{array}$ & $\begin{array}{l}\text { Mengabaikan tugas-tugas } \\
\text { saya }\end{array}$ & $\begin{array}{l}\text { Mengabaikan tugas- } \\
\text { tugas saya }\end{array}$ \\
\hline $\mathrm{C} 43$ & Follow a schedule. & $\begin{array}{l}\text { Melakukan aktivitas } \\
\text { sesuai jadwal atau } \\
\text { agenda }\end{array}$ & $\begin{array}{l}\text { Melaksanakan kegiatan } \\
\text { sesuai dengan jadwal } \\
\text { yang telah direncanakan }\end{array}$ & $\begin{array}{l}\text { Melakukan aktivitas } \\
\text { sesuai jadwal atau } \\
\text { agenda }\end{array}$ \\
\hline $\mathrm{C} 48$ & $\begin{array}{l}\text { Am exacting in my } \\
\text { work. }\end{array}$ & $\begin{array}{l}\text { Sangat telaten dalam } \\
\text { bekerja }\end{array}$ & $\begin{array}{l}\text { Telaten dalam } \\
\text { mengerjakan tugas }\end{array}$ & $\begin{array}{l}\text { Telaten dalam } \\
\text { mengerjakan tugas }\end{array}$ \\
\hline
\end{tabular}


Dimension of emotional stability

The translation of the items in emotional stability dimension can be seen in table 4 . Items on the emotional stability dimension had a lot of translation differences. This was because many terms were quite strange. For example, was item number 44 . The word "irritated" by translator A was interpreted as "jengkel", whereas translator B means "terganggu". When referring to the Oxford dictionary and the familiarity of the word, both translations were equally good, but because the word "terganggu" had been used as a translation of the word "disturbed" in item number 24, the translation was chosen as the word "jengkel".

\section{Dimension of intellect}

The translation of the items in intellect dimension can be seen in table 5. Items in the dimensions of intellect were also relatively easy to translate. However, there were several different translations between the two translators. For example, item number 5, "Have a rich vocabulary" by translator A was translated as "Menguasai banyak perbendaharaan kata", while translator B translated as "Menguasai banyak kosakata". Considering words that were more familiar to Indonesians, finally the final translation chosen was "Menguasai banyak kosakata".

Table 4 .

Translation of items in the emotional stability dimension

\begin{tabular}{|c|c|c|c|c|}
\hline No & Original item & Translation A & Translation B & Final translation \\
\hline EM4 & $\begin{array}{l}\text { Get stressed out } \\
\text { easily. }\end{array}$ & Mudah stres/frustasi & Mudah merasa tertekan & Mudah merasa tertekan \\
\hline EM9 & $\begin{array}{l}\text { Am relaxed } \\
\text { most of the } \\
\text { time. }\end{array}$ & $\begin{array}{l}\text { Merasa tenang hampir } \\
\text { setiap waktu }\end{array}$ & $\begin{array}{l}\text { Merasa tenang hampir } \\
\text { setiap saat }\end{array}$ & $\begin{array}{l}\text { Merasa tenang hampir } \\
\text { setiap saat }\end{array}$ \\
\hline EM14 & $\begin{array}{l}\text { Worry about } \\
\text { things. }\end{array}$ & Mudah khawatir & Mudah khawatir & Mudah khawatir \\
\hline EM19 & $\begin{array}{l}\text { Seldom feel } \\
\text { blue. }\end{array}$ & Jarang merasa sedih & Jarang bersedih & Jarang merasa sedih \\
\hline EM24 & $\begin{array}{l}\text { Am easily } \\
\text { disturbed. }\end{array}$ & $\begin{array}{l}\text { Mudah merasa } \\
\text { terganggu }\end{array}$ & $\begin{array}{l}\text { Mudah merasa } \\
\text { terganggu }\end{array}$ & $\begin{array}{l}\text { Mudah merasa } \\
\text { terganggu }\end{array}$ \\
\hline EM29 & Get upset easily. & Mudah merasa kecewa & Mudah marah & Mudah merasa kesal \\
\hline EM34 & $\begin{array}{l}\text { Change my } \\
\text { mood a lot. }\end{array}$ & $\begin{array}{l}\text { Perasaan saya sering } \\
\text { berubah-ubah }\end{array}$ & $\begin{array}{l}\text { Memiliki perasaan yang } \\
\text { berubah-ubah }\end{array}$ & $\begin{array}{l}\text { Memiliki perasaan yang } \\
\text { berubah-ubah }\end{array}$ \\
\hline EM39 & $\begin{array}{l}\text { Have frequent } \\
\text { mood swings. }\end{array}$ & $\begin{array}{l}\text { Memiliki perasaan yang } \\
\text { mudah berubah }\end{array}$ & $\begin{array}{l}\text { Memiliki suasana hati } \\
\text { yang sering berubah- } \\
\text { ubah }\end{array}$ & $\begin{array}{l}\text { Memiliki suasana hati } \\
\text { yang sering berubah- } \\
\text { ubah }\end{array}$ \\
\hline EM44 & $\begin{array}{l}\text { Get irritated } \\
\text { easily. }\end{array}$ & Mudah merasa jengkel & $\begin{array}{l}\text { Mudah merasa } \\
\text { terganggu }\end{array}$ & Mudah merasa jengkel \\
\hline EM49 & Often feel blue. & Sering merasa sedih & Sering merasa sedih & Sering merasa sedih \\
\hline
\end{tabular}


Table 5.

Translation of items in the intellect dimension

\begin{tabular}{|c|c|c|c|c|}
\hline No & Original item & Translation A & Translation B & Final translation \\
\hline I5 & $\begin{array}{l}\text { Have a rich } \\
\text { vocabulary. }\end{array}$ & $\begin{array}{l}\text { Menguasai banyak } \\
\text { kosakata }\end{array}$ & $\begin{array}{l}\text { Menguasai banyak } \\
\text { perbendaharaan kata }\end{array}$ & $\begin{array}{l}\text { Menguasai banyak } \\
\text { kosakata }\end{array}$ \\
\hline $\mathrm{I} 10$ & $\begin{array}{l}\text { Have difficulty } \\
\text { understanding } \\
\text { abstract ideas. }\end{array}$ & $\begin{array}{l}\text { Mengalami kesulitan } \\
\text { dalam memahami ide-ide } \\
\text { atau hal-hal yang bersifat } \\
\text { abstrak }\end{array}$ & $\begin{array}{l}\text { Kesulitan memahami } \\
\text { ide yang bersifat } \\
\text { abstrak }\end{array}$ & $\begin{array}{l}\text { Kesulitan memahami ide } \\
\text { yang bersifat abstrak }\end{array}$ \\
\hline I15 & $\begin{array}{l}\text { Have a vivid } \\
\text { imagination. }\end{array}$ & $\begin{array}{l}\text { Memiliki imajinasi yang } \\
\text { sangat kuat }\end{array}$ & $\begin{array}{l}\text { Memiliki imajinasi } \\
\text { yang sangat kuat }\end{array}$ & $\begin{array}{l}\text { Memiliki imajinasi yang } \\
\text { sangat kuat }\end{array}$ \\
\hline $\mathrm{I} 20$ & $\begin{array}{l}\text { Am not interested } \\
\text { in abstract ideas. }\end{array}$ & $\begin{array}{l}\text { Tidak tertarik dengan ide- } \\
\text { ide abstrak }\end{array}$ & $\begin{array}{l}\text { Tidak tertarik pada } \\
\text { hal-hal yang bersifat } \\
\text { abstrak }\end{array}$ & $\begin{array}{l}\text { Tidak tertarik dengan } \\
\text { ide-ide abstrak }\end{array}$ \\
\hline $\mathrm{I} 25$ & $\begin{array}{l}\text { Have excellent } \\
\text { ideas. }\end{array}$ & $\begin{array}{l}\text { Memiliki ide-ide yang } \\
\text { cemerlang }\end{array}$ & $\begin{array}{l}\text { Memiliki ide-ide yang } \\
\text { cerdas }\end{array}$ & $\begin{array}{l}\text { Memiliki ide-ide yang } \\
\text { cemerlang }\end{array}$ \\
\hline I30 & $\begin{array}{l}\text { Do not have a } \\
\text { good imagination. }\end{array}$ & $\begin{array}{l}\text { Tidak memiliki imajinasi } \\
\text { yang baik }\end{array}$ & $\begin{array}{l}\text { Tidak memiliki daya } \\
\text { imajinasi yang baik }\end{array}$ & $\begin{array}{l}\text { Tidak memiliki } \\
\text { imajinasi yang baik }\end{array}$ \\
\hline I35 & $\begin{array}{l}\text { Am quick to } \\
\text { understand things. }\end{array}$ & $\begin{array}{l}\text { Cepat dalam memahami } \\
\text { sesuatu }\end{array}$ & $\begin{array}{l}\text { Mudah memahami } \\
\text { suatu hal }\end{array}$ & $\begin{array}{l}\text { Cepat dalam memahami } \\
\text { sesuatu }\end{array}$ \\
\hline $\mathrm{I} 40$ & $\begin{array}{l}\text { Use difficult } \\
\text { words. }\end{array}$ & $\begin{array}{l}\text { Mahir menggunakan } \\
\text { istilah-istilah sulit dalam } \\
\text { menyampaikan sesuatu } \\
\text { baik lisan maupun tulisan }\end{array}$ & $\begin{array}{l}\text { Menggunakan istilah- } \\
\text { istilah yang sulit }\end{array}$ & $\begin{array}{l}\text { Menggunakan istilah- } \\
\text { istilah yang sulit }\end{array}$ \\
\hline $\mathrm{I} 45$ & $\begin{array}{l}\text { Spend time } \\
\text { reflecting on } \\
\text { things. }\end{array}$ & $\begin{array}{l}\text { Meluangkan waktu untuk } \\
\text { merefleksi hal atau } \\
\text { peristiwa }\end{array}$ & $\begin{array}{l}\text { Meluangkan waktu } \\
\text { untuk merefleksikan } \\
\text { berbagai hal }\end{array}$ & $\begin{array}{l}\text { Meluangkan waktu } \\
\text { untuk merefleksikan } \\
\text { berbagai hal }\end{array}$ \\
\hline I50 & Am full of ideas. & $\begin{array}{l}\text { Memiliki banyak gagasan } \\
\text { atau ide }\end{array}$ & Memiliki banyak ide & Memiliki banyak ide \\
\hline
\end{tabular}

\section{Back translation}

The results of the back translation by language professionals were consulted with scale developer via email. From the results of consultations with the developer, it was concluded that all items had the same meaning as the original item. In addition, the back translation items were also able to measure the same constructs as intended in the measuring objectives.

\section{Translation review}

The translations review were carried out by three lecturers of the Faculty of Psychology UGM who had experience in translating and had lived in an English speaking country for at least two years. From the results of the review, there were minor revisions on items number 23, 32, and 39.

\section{Readability test}

Readability tests were conducted on December 20-24, 2017 to five people, consisting of 2 entrepreneurs, 1 high school student, and 2 Psychology students. All respondents said that all items on the scale could already be understood.

\section{Content validity}

The items in IPIP-BFM-50 were given to the rater to be assessed how relevant the item related to the dimensions measured. The 
raters in this study were 12 people consisting of 11 Psychology Postgraduate students and one lecturer of Psychology with a range of scores $1-5$. Considering the rater number (12 raters), an item was determined to be relevant only if it had a minimum Aiken $\mathrm{V}$ content validity index of 0.69 (Aiken, 1985). Of the 50 items, the index value ranged between 0.71 to 0.98 . Thus, all of the items in the IPIP-BFM-50 translation had met the criteria and were declared as a valid item to measure the five dimensions.

Stage 2: Evaluation of psychometric properties of Indonesian IPIP-BFM-50

\section{Item-total correlation and reliability}

The item-total correlation value and reliability of the scale were estimated by a sample of 115 people aged ranging from 1650 years. The number of male subjects was 28 people $(24.3 \%)$, while the female subjects were 87 people $(75.7 \%)$. The internal consistency was seen from the corrected item-total correlation in each dimension. Reliability was seen from the value of alpha reliability coefficient. The scale summary is shown in table 6 .

On an extraversion dimension, the item-total correlation value ranges from 0.527 to 0.741 with an alpha reliability coefficient of 0.87 . On the agreeableness dimension, the item-total correlation value ranges from $0.355-0.673$ with an alpha reliability coefficient of 0.83 . On the conscientiousness dimension, the itemtotal correlation value ranges from 0.376 0.597 with alpha reliability coefficient 0.81 . On the scale of emotional stability dimension, the item-total correlation value ranges from $0.489-0.666$ with an alpha reliability coefficient of 0.86 . On the intellect dimension, the total item correlation value ranges from $0.273-0.698$ with an alpha reliability coefficient of 0.81 .

Table 6 indicates that all items have an item-total correlation above the standard, which is 0.25 (Kline, 1986). It shows that all items in the Indonesian IPIP-BFM-50 scale have a good function to distinguish between individuals who have and do not have measurable attributes. In addition, all scales have alpha reliability above 0.70 , thus it could be concluded that each scale has satisfactory reliability, so the measurement results with IPIP-BFM-50 could be trusted.

\section{Factor analysis}

Before factor analysis was conducted, reliability testing was implemented with a sample of 502 people. The reliability of the scales were as follows: Extraversion $\alpha=$ 0.836, Agreeableness $\alpha=0.762$, Conscientiousness $\alpha=0.811$, Emotional stability $\alpha=$ 0.862 , and Intellect $\alpha=0.768$. Factor analysis was carried out on 502 respondents.

Table 6.

Item-total correlation and reliability of Indonesian BFM-50 IPIP ( $\mathrm{n}=115$ )

\begin{tabular}{lcccc}
\hline \multicolumn{1}{c}{ Dimension } & Number of items & Item-total correlation & Alpha & SEM \\
\hline Extraversion & 10 & $0,527-0,741$ & 0,87 & 2,51 \\
Agreeableness & 10 & $0,355-0,673$ & 0,83 & 2,34 \\
Conscientiousness & 10 & $0,376-0,597$ & 0,81 & 2,69 \\
Emotional stability & 10 & $0,489-0,666$ & 0,86 & 2,54 \\
Intellect & 10 & $0,273-0,698$ & 0,81 & 2,48 \\
\hline
\end{tabular}

Note: SEM= Standard Error of Measurement 
Factor analysis was conducted to see the distribution of items in accordance with the blueprint. Stevens (1992) recommended that items that have a loading factor above 0.4 were feasible to maintain. The results showed the value of the Barlett Test of Sphericity was 9136,419 with $\mathrm{p}<0.01$ which means there was a significant correlation among the variables. The KMO 0.828 indicated that factor analysis could be continued. The distribution of the loading factors of each item to the dimensions measured is shown in table 7 below.

Table 7.

Rotated factors of IPIP-BFM-50 ( $\mathrm{n}=502)$

\begin{tabular}{lccccc}
\hline \multirow{2}{*}{ Item } & \multicolumn{5}{c}{ Factor } \\
\cline { 2 - 6 } & $\mathbf{1}$ & $\mathbf{2}$ & $\mathbf{3}$ & $\mathbf{4}$ & $\mathbf{5}$ \\
\hline ES39 & $\mathbf{0 , 7 3}$ & 0,08 & 0,12 & $-0,03$ & 0,06 \\
ES44 & $\mathbf{0 , 7 3}$ & $-0,05$ & 0,04 & 0,23 & 0,10 \\
ES14 & $\mathbf{0 , 6 9}$ & 0,14 & $-0,07$ & $-0,05$ & $-0,02$ \\
ES29 & $\mathbf{0 , 6 8}$ & $-0,07$ & 0,10 & 0,22 & 0,11 \\
ES49 & $\mathbf{0 , 6 8}$ & 0,13 & 0,09 & $-0,01$ & $-0,07$ \\
ES4 & $\mathbf{0 , 6 8}$ & 0,12 & 0,02 & $-0,00$ & $-0,06$ \\
ES34 & $\mathbf{0 , 6 7}$ & 0,02 & 0,12 & $-0,03$ & 0,07 \\
ES24 & $\mathbf{0 , 6 1}$ & 0,05 & $-0,12$ & 0,21 & 0,09 \\
ES19 & $\mathbf{0 , 5 3}$ & 0,15 & 0,11 & $-0,01$ & $-0,07$ \\
ES9 & $\mathbf{0 , 4 1}$ & $-0,05$ & 0,10 & 0,10 & 0,12 \\
E31 & 0,10 & $\mathbf{0 , 7 5}$ & 0,11 & 0,14 & 0,04 \\
E26 & 0,03 & $\mathbf{0 , 6 9}$ & $-0,10$ & $-0,05$ & 0,02 \\
E6 & 0,03 & $\mathbf{0 , 6 8}$ & $-0,08$ & $-0,09$ & 0,05 \\
E1 & 0,06 & $\mathbf{0 , 6 5}$ & 0,03 & 0,11 & 0,03 \\
E21 & 0,05 & $\mathbf{0 , 6 4}$ & 0,12 & 0,16 & 0,06 \\
E36 & 0,00 & $\mathbf{0 , 6 4}$ & $-0,05$ & $-0,05$ & 0,16 \\
E41 & $-0,05$ & $\mathbf{0 , 6 0}$ & 0,00 & 0,05 & 0,12 \\
E16 & 0,07 & $\mathbf{0 , 5 4}$ & $-0,07$ & $-0,02$ & $-0,02$ \\
E46 & 0,09 & $\mathbf{0 , 5 3}$ & 0,01 & 0,13 & 0,06 \\
E11 & 0,08 & $\mathbf{0 , 4 9}$ & 0,00 & 0,30 & $-0,05$ \\
C3 & 0,01 & 0,01 & $\mathbf{0 , 7 0}$ & 0,06 & 0,03 \\
C33 & $-0,14$ & $-0,03$ & $\mathbf{0 , 6 9}$ & 0,03 & 0,01 \\
C43 & $-0,00$ & 0,01 & $\mathbf{0 , 6 8}$ & 0,08 & 0,04 \\
C23 & 0,06 & 0,03 & $\mathbf{0 , 6 5}$ & 0,10 & $-0,02$ \\
C48 & 0,10 & $-0,09$ & $\mathbf{0 , 6 3}$ & 0,17 & 0,17 \\
C13 & $-0,11$ & $-0,01$ & $\mathbf{0 , 5 7}$ & 0,11 & 0,26 \\
C38 & 0,21 & 0,00 & $\mathbf{0 , 5 1}$ & 0,18 & $-0,03$ \\
C8 & 0,23 & $-0,04$ & $\mathbf{0 , 4 7}$ & $-0,01$ & $-0,05$ \\
C28 & 0,35 & $-0,01$ & $\mathbf{0 , 4 7}$ & $-0,07$ & 0,11 \\
C18 & 0,31 & 0,08 & $\mathbf{0 , 4 6}$ & 0,17 & $-0,13$ \\
\hline A7 & 0,00 & 0,13 & 0,11 & $\mathbf{0 , 7 0}$ & 0,01
\end{tabular}

\begin{tabular}{lccccc}
\hline \multirow{2}{*}{ Item } & \multicolumn{5}{c}{ Factor } \\
\cline { 2 - 6 } & $\mathbf{1}$ & $\mathbf{2}$ & $\mathbf{3}$ & $\mathbf{4}$ & $\mathbf{5}$ \\
\hline A37 & 0,02 & 0,10 & 0,09 & $\mathbf{0 , 6 5}$ & 0,08 \\
A17 & $-0,02$ & 0,00 & 0,14 & $\mathbf{0 , 6 4}$ & 0,10 \\
A42 & 0,03 & 0,16 & 0,15 & $\mathbf{0 , 6 4}$ & 0,12 \\
A47 & 0,04 & 0,20 & 0,24 & $\mathbf{0 , 5 6}$ & 0,11 \\
A32 & 0,09 & 0,31 & $-0,06$ & $\mathbf{0 , 5 2}$ & 0,03 \\
A12 & 0,27 & $-0,14$ & 0,24 & $\mathbf{0 , 4 7}$ & $-0,06$ \\
A22 & 0,05 & 0,20 & $-0,18$ & $\mathbf{0 , 4 3}$ & 0,00 \\
A27 & 0,10 & $-0,22$ & 0,25 & $\mathbf{0 , 4 1}$ & $-0,02$ \\
A2 & 0,03 & $-0,09$ & 0,03 & $\mathbf{0 , 4 0}$ & 0,07 \\
I50 & 0,06 & 0,08 & 0,05 & 0,15 & $\mathbf{0 , 7 4}$ \\
I15 & $-0,15$ & 0,04 & $-0,04$ & 0,17 & $\mathbf{0 , 6 7}$ \\
I30 & 0,19 & 0,07 & $-0,02$ & 0,15 & $\mathbf{0 , 6 1}$ \\
I25 & 0,06 & 0,21 & 0,12 & 0,17 & $\mathbf{0 , 6 1}$ \\
I10 & 0,22 & 0,00 & $-0,03$ & $-0,07$ & $\mathbf{0 , 5 5}$ \\
I20 & 0,07 & 0,00 & $-0,03$ & 0,05 & $\mathbf{0 , 5 5}$ \\
I35 & 0,07 & 0,09 & 0,28 & 0,13 & $\mathbf{0 , 5 1}$ \\
I5 & $-0,10$ & 0,17 & 0,13 & $-0,10$ & $\mathbf{0 , 4 9}$ \\
I40 & $-0,13$ & 0,00 & $-0,06$ & $-0,14$ & $\mathbf{0 , 4 3}$ \\
I45 & 0,06 & $-0,08$ & 0,23 & 0,23 & $\mathbf{0 , 3 4}$ \\
\hline
\end{tabular}

Table 7 indicates that item number 45 has a loading factor below 0.4. However, the item is well distributed in accordance with the blueprint which is in the intellect dimension group with a value of 0.348 . The other items are well distributed according to the expected dimension and have a loading factor above 0.4 . It shows that the IPIP-BFM-50 correctly measures the five big five dimensions. Thus, it can be concluded that this scale has a good construct validity

\section{Discussion}

The purpose of this study is to adapt the IPIP-BFM-50 into Indonesian. Therefore there is an alternative instrument measuring big five personality that is open to the public and has satisfying psychometric properties. From the results of this adaptation study it was found that the scale of IPIP-BFM-50 which had been translated into Indonesian was easily understood by respondents and had 
satisfactory psychometric properties. Alpha reliability values of Extraversion $\alpha=$ 0.836, Agreeableness $\alpha=0.762$, Conscientiousness $\alpha=0.811$, Emotional stability $\alpha=$ 0.862 , and Intellect $\alpha=0.768$. This value has met the minimum reliability coefficient suggested by Nunnally (1978) which is above 0.7 .

In adaptation procedures, many adaptation guidelines emphasize that the final translation should not only consider the equality of translations with the original language, but also by looking at the context of local culture (Azwar, 2017; Beaton et al., 2000; International Test Commission, 2016). This is also considered in the adaptation procedure of IPIP-BFM-50. There is the word "party" in item 1 and 31 which is literally translated into Indonesian as "pesta". However, by looking at the culture of the Indonesian who are not familiar with parties like in the Western, the word "party" in this study is translated more generally as "acara". Thus respondents can be more flexible when asked to assess themselves.

When compared with other instrument measuring Big Five personality traits using Indonesian language (BFI), the reliability coefficient of IPIP-BFM Indonesia is greater. In the Ramdhani (2012) study it was reported that Alpha reliability of items selected ranged from 0.73 - 0.79 . Meanwhile, the Alpha reliability of IPIPBFM Indonesia in the subjects of 502 people ranged from $0.762-0.862$. In addition, the items in IPIP-BFM Indonesia are also easily understood by respondents because they are brief and straightforward. The terms used in items are quite familiar to most Indonesians.

When compared with the previous study from Maharani (2013) which had the same purpose, the translation results from this study also had better psychometric properties. In Maharani's (2013) study, the value of Alpha reliability ranged from 0.587 - 0.824, while Alpha reliability in this study ranged from 0.762 - 0.862 . In addition, the results of the adaptation scale factor analysis in Maharani's (2013) study were also unsatisfactory. Some items have a low loading factor in their dimensions and have a high cross-loading. This does not happen in this study, because each item is distributed according to the dimension and almost all of the items have a loading factor above 0.4 .

Based on the reliability estimation, this study has similar results as the study of Strus et al., (2017) who conducted adaptation on the Polish version of IPIP-BFM-50. The reliability coefficient value in the intellect dimension is relatively low when compared to the other dimensions. Similarly, the results of factor analysis also have similar findings with the study of Topolewska et al., (2017) on the Polish version of the IPIP-BFM-50. Items number 45 have a relatively low loading factor. Item number 45 ("meluangkan waktu untuk merefleksikan berbagai hal") has a fairly high cross-loading in the dimensions of agreeableness and conscientiousness. The word "merefleksikan" is quite ambiguous because it often overlaps with "perencanaan" which is very closely related to the conscientiousness dimension. Besides that, the word " meluangkan waktu" is closely related to "pengorbanan" which is related to the dimensions of agreeableness.

The output of this study is a big five personality inventory that is valid and reliable and suitable for the culture of Indonesian society. The existence of alternative big five personality inventory in the Indonesian language hopefully can improve the research on personality. In addition, the standard format of IPIP-BFM and the popularity of IPIP-BFM in various 
countries make this scale and can be used for cross-country study of personality. The character of IPIP-BFM-50 which is open source also allows this scale to be used for various purposes easily, both for commercial and non-commercial purposes.

Various future developments related to personality measurement are certainly still required. In this study, a validation procedure was carried out through content validity and exploratory factor analysis. In the future, several validation procedures are also required, such as with Confirmatory Factor Analysis and criterion validity. In addition, because the sample of this study was limited to the Java region, in the future this scale needs to be piloted to a larger number of samples outside Java so that it can be known whether this scale fits the local culture.

IPIP-BFM-50 was basically compiled for research purposes, not for individual diagnosis. So, both scale developers and researchers did not formulate the norms of IPIP-BFM-50. But with the advantages of IPIP-BFM-50 which is open and can be used freely by researchers, the development of the norm of this scale can be considered. The development of the norm can be achieved through the collaboration of various personality researchers in Indonesia. With the existence of norms, the relative position of individuals towards Indonesian society groups can be known. In addition, the existence of state norms can also be useful if used for cross-country personality studies.

\section{Conclusion}

Based on the adaptation process of IPIPBFM-50 into Indonesia, it is concluded that all items in the IPIP-BFM-50 have item-total correlations above 0.25 and the five scales in IPIP-BFM-50 have alpha reliability above
0.7. The results of the factor analysis also indicate that there are five main factors with existing items well distributed according to their dimensions. Thus, the scale of IPIP-BFM-50 has satisfactory validity and reliability. IPIP-BFM- 50 can be an alternative personality measurement instrument that is open-source and can be used for various purposes, especially for personality research.

\section{Recommendation}

In order to prove that IPIP-BFM-50 can be applied to measure the personality of a wider Indonesian society, further researchers are advised to reevaluate the psychometric properties of IPIP-BFM-50 in samples outside Java as well as with other validation procedures, such as Confirmatory Factor Analysis and criterion validity.

\section{References}

Aiken, L. R. (1985). Three coefficients for analyzing the reliability and validity of ratings. Educational and Psychological Measurement, 45(1), 131-142. doi: 10.1177/0013164485451012

Azwar, S. (2012). Penyusunan skala psikologi (2nd ed.). Yogyakarta: Pustaka Pelajar.

Azwar, S. (2017). Metode penelitian psikologi (2nd ed.). Yogyakarta: Pustaka Pelajar.

Beaton, D. E., Bombardier, C., Guillemin, F., \& Ferraz, M. B. (2000). Guidelines for the process of cross-cultural adaptation of self-report measures. Spine, 25(24), 3186-3191.

Costa Jr, P. T., \& McCrae, R. R. (1995). Domains and facets: Hierarchical personality assessment using the Revised NEO Personality Inventory. Journal of Personality Assessment, 64(1), 
21-50. doi: 10.1207/s15327752jpa 6401_2

Donnellan, M. B., Oswald, F. L., Baird, B. M., \& Lucas, R. E. (2006). The Mini-IPIP Scales: Tiny-yet-effective measures of the Big Five Factors of Personality. Psychological Assessment, 18(2), 192-203. doi: 10.1037/1040-3590.18.2.192

Goldberg, L. R. (1992). The development of markers for the Big-Five factor structure. Psychological Assessment, 4(1), 26. doi: 10.1037/1040-3590.4.1.26

Goldberg, L. R., Johnson, J. A., Eber, H. W., Hogan, R., Ashton, M. C., Cloninger, C. R., \& Gough, H. G. (2006). The international personality item pool and the future of public-domain personality measures. Journal of Research in Personality, 40(1), 84-96. doi: 10.1016/j.jrp.2005.08.007

Gow, A. J., Whiteman, M. C., Pattie, A., \& Deary, I. J. (2005). Goldberg's "IPIP" Big-Five factor markers: Internal consistency and concurrent validation in Scotland. Personality and Individual Differences, 39(2), 317-329. doi: 10.1016/j.paid.2005.01.011

International Test Commission. (2016). The ITC guidelines for translating and adapting tests (second edition). Retrieved from www.InTestCom.org

John, O. P., \& Srivastava, S. (1999). The big five trait taxonomy: History, measurement, and theoretical perspectives. Handbook of Personality: Theory and Research, 2, 102-138.

Kline, P. (1986). A handbook of test construction: Introduction to psychometric design. London: Methuen.
Maharani, R. F. (2013). Adaptasi alat ukur kepribadian dari International Personality Item Pool (IPIP). Skripsi (unpublished). Fakultas Psikologi Universitas Sumatera Utara.

Mlačić, B., \& Goldberg, L. R. (2007). An analysis of a cross-cultural personality inventory: The IPIP Big-Five Factor Markers in Croatia. Journal of Personality Assessment, 88(2), 168-177. doi: $10.1080 / 00223890701267993$

Nunnally, J. (1978). Psychometric theory. 2nd Edition. New York: McGraw-Hill.

Ramdhani, N. (2012). Adaptasi bahasa dan budaya dari skala kepribadian Big Five. Jurnal Psikologi, 39(2), 189-205. 10.22146/jpsi.6986

Stevens, J. P. (1992). Applied multivariate statistics for the social sciences (2nd edition). Hillsdale, NJ: Erlbaum.

Strus, W., Cieciuch, J., \& Rowiński, T. (2017). The Polish adaptation of the IPIP-BFM-50 questionnaire for measuring five personality traits in the lexical approach. Roczniki Psychologiczne/Annals of Psychology, 17(2), 347-366.

Topolewska, E., Skimina, E., Strus, W., Cieciuch, J., \& Rowiński, T. (2017). The short IPIP-BFM-20 questionnaire for measuring the Big Five. Roczniki Psychologiczne/Annals of Psychology, 17(2), 385-402.

Ypofanti, M., Zisi, V., Zourbanos, N., Mouchtouri, B., Tzanne, P., Theodorakis, Y., \& Lyrakos, G. (2015). Psychometric properties of the International Personality item Pool Big-Five personality questionnaire for the Greek population. Health Psychology Research, 3(2). doi: 10.4081/hpr.2015.2206 